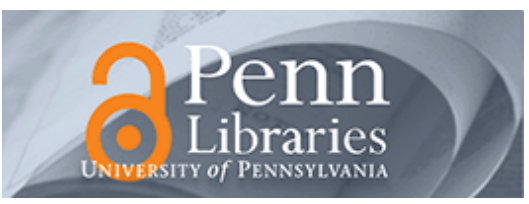

University of Pennsylvania ScholarlyCommons

\title{
Fractionating the Left Frontal Response to Tools: Dissociable Effects of Motor Experience and Lexical Competition
}

Irene P Kan

University of Pennsylvania

Joseph W. Kable

University of Pennsylvania, kable@psych.upenn.edu

Amanda Van Scoyoc

University of Pennsylvania

Anjan Chatterjee

University of Pennsylvania

Sharon L. Thompson-Schill

University of Pennsylvania, sschill@psych.upenn.edu

Follow this and additional works at: https://repository.upenn.edu/psychology_papers

Part of the Psychology Commons

\section{Recommended Citation}

Kan, I., Kable, J. W., Van Scoyoc, A., Chatterjee, A., \& Thompson-Schill, S. L. (2006). Fractionating the Left Frontal Response to Tools: Dissociable Effects of Motor Experience and Lexical Competition. Journal of Cognitive Neuroscience, 18 (2), 267-277. http://dx.doi.org/10.1162/jocn.2006.18.2.267

This paper is posted at ScholarlyCommons. https://repository.upenn.edu/psychology_papers/21

For more information, please contact repository@pobox.upenn.edu. 


\title{
Fractionating the Left Frontal Response to Tools: Dissociable Effects of Motor Experience and Lexical Competition
}

\author{
Abstract \\ A number of theories about the evolution of language posit a close (and perhaps causal) relationship \\ between tool use and speech. Consistent with this idea, neuroimaging studies have found that tool \\ knowledge retrieval activates not only a region of the left premotor cortex involved in hand action, but \\ also an adjacent region that is typically described as a language center. We examined whether this \\ pattern of activation is best described as the result of a single process, related to both action and \\ language, or the result of two independent processes. We identified two distinct neural components that \\ jointly contribute to this response: a posterior region centered in the premotor cortex, which responds to \\ motor knowledge retrieval, and an anterior region centered in the left frontal operculum, which responds \\ to lexical competition. Crucial to the interpretation of the premotor response, individual variation in motor \\ experience was highly correlated with the magnitude of the response in the premotor cortex, but not in \\ the prefrontal cortex. \\ Disciplines \\ Psychology
}




\title{
Fractionating the Left Frontal Response to Tools: Dissociable Effects of Motor Experience and Lexical Competition
}

\author{
Irene P. Kan, Joseph W. Kable, Amanda Van Scoyoc, Anjan Chatterjee, \\ and Sharon L. Thompson-Schill
}

\begin{abstract}
A number of theories about the evolution of language posit a close (and perhaps causal) relationship between tool use and speech. Consistent with this idea, neuroimaging studies have found that tool knowledge retrieval activates not only a region of the left premotor cortex involved in hand action, but also an adjacent region that is typically described as a language center. We examined whether this pattern of activation is best described as the result of a single process, related to both action and language, or the result of two
\end{abstract}

\section{INTRODUCTION}

According to one prominent theory of conceptual knowledge organization (Allport, 1985), a strong relationship exists between our sensorimotor experiences and our conceptual and neural representations. Specifically, Allport (1985) proposed that conceptual knowledge is organized as a pattern of auto-associated activity distributed across different sensory (e.g., visual, tactile, auditory) and motor (e.g., action, kinesthetic) domains. Furthermore, under this account, information is stored in or near brain areas that are recruited when acquiring that knowledge. That is, whereas visually acquired information about "hammer" is stored in or near visual areas, action-oriented information is stored in or near motor areas. Thus, the representation of hammer is distributed across different domain attributes, and when the concept hammer is retrieved, a distributed pattern of associated neural activity is expected.

Support for a distributed sensorimotor account of conceptual knowledge has come from a number of recent neuroimaging studies in which recruitment of a distributed network of brain areas during the identification of manmade tools has been reported (e.g., Tyler et al., 2003; Chao \& Martin, 2000; Perani et al., 1999; Grabowski, Damasio, \& Damasio, 1998; Grafton, Fadiga, Arbib, \&

University of Pennsylvania independent processes. We identified two distinct neural components that jointly contribute to this response: a posterior region centered in the premotor cortex, which responds to motor knowledge retrieval, and an anterior region centered in the left frontal operculum, which responds to lexical competition. Crucial to the interpretation of the premotor response, individual variation in motor experience was highly correlated with the magnitude of the response in the premotor cortex, but not in the prefrontal cortex.

Rizzolatti, 1997; Martin, Wiggs, Ungerleider, \& Haxby, 1996). For example, Martin et al. (1996) asked subjects to name a series of black and white line drawings of tools and animals and observed tool-specific PET activation in the left middle temporal gyrus, the right supramarginal gyrus, and the left ventrolateral frontal cortex, extending from the frontal operculum (Brodmann's area [BA] 44/ 45) to the ventrolateral premotor cortex (vLPM, BA 6). Because the distributed network recruited during tool naming included regions that have been implicated in action and motion processing (left middle temporal gyrus, e.g., Martin, Haxby, Lalonde, Wiggs, \& Ungerleider, 1995 ) and motor imagery and execution (left ventrolateral premotor cortex, e.g., Decety, 1996), the authors argued that these data reflect the sensory-motor nature of tool representations and are consistent with a distributed model of conceptual knowledge organization.

Because vLPM and the left frontal operculum are often coactivated in tasks that involved action and motor knowledge retrieval, this left ventrolateral frontal region has been linked to storage of motor and action knowledge (e.g., Martin, 2001; Martin et al., 1996). However, there are reasons to believe that these two cytoarchitecturally distinct regions within the left ventrolateral frontal cortex subserve different functions (Rizzolatti, Fogassi, \& Gallese, 2002). First, if both regions are involved in motor knowledge retrieval, we should observe recruitment of both areas in every instance when motor knowledge is accessed. However, that is not the case: 
Whereas vLPM has consistently been linked to motor knowledge retrieval, involvement of the left frontal operculum in motor knowledge retrieval has been less consistent. Second, the left frontal operculum has been implicated in a more general mechanism of cognitive control, such as selection among competing alternatives (e.g., Thompson-Schill, D'Esposito, Aguirre, \& Farah, 1997; for different proposals, see Bunge, Kahn, Wallis, Miller, \& Wagner, 2003; Wagner, Pare-Blagoev, Clark, \& Poldrack, 2001). In the present article, we will briefly review some recent evidence that supports each of these claims. Furthermore, we will explore whether the "tool-specific activation" observed throughout left ventrolateral frontal cortex is best thought of as the result of a single process (i.e., motor knowledge retrieval) or of two independent processes (i.e., motor knowledge retrieval and selection among competing alternatives).

Activation in VLPM has been observed in a number of neuroimaging studies that require either implicit retrieval (e.g., tool identification: Chao \& Martin, 2000; Perani et al., 1999; Grabowski et al., 1998; Grafton et al., 1997; Martin et al., 1996; tool name generation: Vitali et al., 2005) or explicit retrieval of action knowledge (e.g., verb reading: Grezes, Tucker, Armony, Ellis, \& Passingham, 2003; Kellenbach, Brett, \& Patterson, 2003; Kable, Lease-Spellmeyer, \& Chatterjee, 2002; Martin et al., 1995; action-related sentence comprehension: Tettamanti et al., 2005; for reviews, see Johnson-Frey, 2003; Pulvermuller, 1999). For example, Hauk, Johnsrude, and Pulvermuller (2004) reported increased activity in premotor cortex when subjects read action words (e.g., kick). Moreover, the authors found that activations along premotor areas were differentially sensitive to action words that refer to different body parts (e.g., lick, pick, kick), such that activations were in or near brain regions involved in actual movements of the tongue, fingers, and feet. The relation between hand action and tool knowledge, in particular, is supported by the consistent observation of overlapping activations within the premotor cortex during imagined grasping and tool knowledge retrieval (e.g., Decety, 1996; for a review, see Grezes \& Decety, 2001). This association has led some researchers to argue that knowledge of tool use and manipulation is stored in vLPM (e.g., Martin, 2001).

Although vLPM activity during identification of manipulable objects has been consistently observed across many studies, recruitment of the left frontal operculum during tool identification has been less consistent (Kellenbach et al., 2003; Kable et al., 2002; Phillips, Noppeney, Humphreys, \& Price, 2002; Chao \& Martin, 2000). For example, in a functional magnetic resonance imaging (fMRI) study, Chao and Martin (2000) reported greater activity in vLPM for tools than animals, but activity in the left frontal operculum was undifferentiated between tool identification and animal identifica- tion. In order to explain the null effect in the left frontal operculum, the authors proposed that whereas vLPM activity is associated with retrieval of the motor aspect of tool use knowledge, the left frontal operculum activity may reflect lexical retrieval and phonological processing. Because lexical retrieval and phonological processing requirements were similar across the two object categories, activity in the left frontal operculum did not differ across the two conditions.

Previous neuroimaging and neuropsychological studies have implicated the left frontal operculum in a domaingeneral cognitive control mechanism that is engaged when one must select an appropriate representation or response among competing alternatives (i.e., the number of competing alternatives for any given situation, Kan \& Thompson-Schill, 2004b; Thompson-Schill, 2003; Thompson-Schill et al., 2002; Bunge, Ochsner, Desmond, Glover, \& Gabrieli, 2001; Barch, Braver, Sabb, \& Noll, 2000; Buckner, Koutstaal, Schacter, \& Rosen, 2000; ThompsonSchill, D'Esposito, \& Kan, 1999; Desmond, Gabrieli, \& Glover, 1998; Thompson-Schill et al., 1998; ThompsonSchill et al., 1997). In the context of object identification, one source of competition may come from the number of name alternatives applicable to a single object. For example, whereas a picture of an orange may evoke only one name (i.e., high name agreement), a picture of a couch may also elicit other name alternatives such as sofa and loveseat (i.e., low name agreement). We have previously demonstrated that activity in the left frontal operculum was modulated by the extent to which a picture evokes a single, reliable name (Kan \& Thompson-Schill, 2004a). That is, identification of low name agreement pictures (i.e., high competition condition, with higher selection demands) produced increased left frontal operculum activity compared to high name agreement pictures (i.e., low competition condition, with lower selection demands). As such, picture name agreement may serve as a valid index of selection demands and the degree of competition present in picture naming.

In the present experiment, we provide a direct test of functional dissociations between vLPM and the left frontal operculum. We hypothesize that previously observed tool-specific activations in the left ventrolateral frontal cortex may reflect two distinct processes: selection among competing conceptual and lexical representations in the left frontal operculum and retrieval of motor knowledge associated with tool use in vLPM. As proposed by Sternberg $(2001,2004)$, in order to demonstrate functional dissociations, it is absolutely critical to demonstrate that each region is selectively influenced by different experimental factors. In other words, it is not enough to show that manipulation of one factor (e.g., object category: tools vs. animals) exerts a significant influence in one region (e.g., vLPM) and not in another region (e.g., left frontal operculum) because a null effect could reflect either low statistical power or variability. To argue for functional dissociations, we 
demonstrate that vLPM and the left frontal operculum are differentially sensitive to different experimental manipulations (i.e., motor knowledge retrieval vs. selection among competing alternatives).

A secondary purpose of this experiment was to further explore the idea that motor experiences have a direct impact on neural representations of object-concepts, as proposed by Allport (1985). If a relationship exists between motor experience with manipulable objects and neural representations of those object-concepts, we would expect different motor experiences to result in different neural representations of those objects. One rudimentary way to quantify variability in motor experiences across individuals is by evaluating hand preference in object use. It seems reasonable to assume that righthanders are more likely to interact with objects with their right hand, and left-handers are more likely to interact with objects using their left hand. Thus, individuals in these two groups may have differential motor experiences associated with their dominant hands. If information is stored in or near brain areas that are recruited when acquiring knowledge, we might expect motor knowledge acquired with the right hand to be stored in the vLPM near the left motor cortex and motor knowledge acquired with the left hand to be stored in the right ventrolateral premotor area (vRPM) near the right motor cortex. To date, most studies that have examined the neural bases of conceptual knowledge have limited their subject population to righthanders only. In this experiment, we explore the impact of motor experience on conceptual representations in both left-handers and right-handers. If vLPM activity during motor knowledge retrieval reflects motor experience with the right hand, we should observe modulation of vLPM activity as a function of right-hand use in object manipulation. Furthermore, we may observe increased involvement of the vRPM for individuals who have relatively more experience in manipulating objects with their left hand.

We used fMRI to examine neural activity associated with identification of animals and manipulable objects in 16 individuals (8 left-handers and 8 right-handers). Selection demands and degree of competition were manipulated by systematically varying picture name agreement (i.e., higher name agreement reflects lower competition and selection demands, and lower name agreement reflects higher competition and selection demands). Black-and-white photographs from each of the four conditions (i.e., low-competition animals, highcompetition animals, low-competition objects, and high-competition objects) were presented in blocks of six trials, and subjects were asked to name the pictures aloud into a fiber optic microphone. Alternating with each experimental block was a block of baseline trials, which consisted of a simple perceptual judgment task that required a yes/no response (see Figure 1A and Methods).

\section{RESULTS}

\section{Behavioral Results from Norming Study}

In a norming study, a group of 32 independent subjects were shown a total of 207 black-and-white photographs and were asked to name each picture. Responses from this phase were used to derive name agreement level for each item and to facilitate item selection for the experiment. To determine name agreement for each item, we tabulated the name responses across individuals and selected the response with the highest count, and the name agreement index was calculated as the percentage of participants who agree on that name. Furthermore, the variability in name agreement across subjects was used as a proxy measure of the degree of lexical competition within individuals. Based on these results, a final set of 144 photographs was selected. For the final set of 144 photographs used in the experiment, the mean name agreement for low competition items was 95.8\% (range 88-100\%) and the mean name agreement for high competition items was $63.4 \%$ (range 34-88\%). An unpaired $t$ test revealed a significant difference in name agreement percentages between the two sets of photographs, $t(142)=18.97, p<.001$. Furthermore, within each level of competition, there was no difference in mean name agreement between the two object categories: low-competition animals $=95.8 \%, S D=3.9 \%$ and low-competition objects $=96.2 \%, S D=3.9 \%, t(70)=$ $-0.38, p=n s$; high-competition animals $=64.1 \%, S D=$ $13.8 \%$ and high-competition objects $=62.8 \%, S D=14.6 \%$, $t(70)=0.38, p=n s$.

In the second phase of the norming study, 12 additional subjects named the set of 144 photographs, one at a time, and their response times were collected. Across subjects, mean reaction times and standard deviation for the four conditions were as follows: low-competition animals $=996 \mathrm{msec}, S D=129 \mathrm{msec}$; low-competition objects $=931 \mathrm{msec}, S D=111 \mathrm{msec}$; high-competition animals $=1162 \mathrm{msec}, S D=130 \mathrm{msec}$; high-competition objects $=1099 \mathrm{msec}, S D=129 \mathrm{msec}$. A 2 (category) $\times 2$ (competition) ANOVA revealed a significant category main effect, $F(1,11)=28.26$, $p<.01$, and a significant competition main effect, $F(1,11)=6.50, p=.03$. The interaction was not significant $(F<1.0, p=n s)$. In sum, response times and name agreement percentages were matched across the two categories (animals vs. objects) within each level of competition.

\section{Behavioral Results from Scanner}

Subjects' responses were compared to the names collected in the norming study, and a response (e.g., hammer) was considered a match if it was the most common name given during pilot testing. Overall agreement percentages across subjects were then calculated. A paired $t$ test of subjects' response agreement revealed 
Figure 1. Trial blocking, timing, and composition. (A) Pictorial depiction of experimental and baseline conditions. Eight counterbalancing versions were used to control for presentation order across subjects. (B) Trial timing and trial composition.

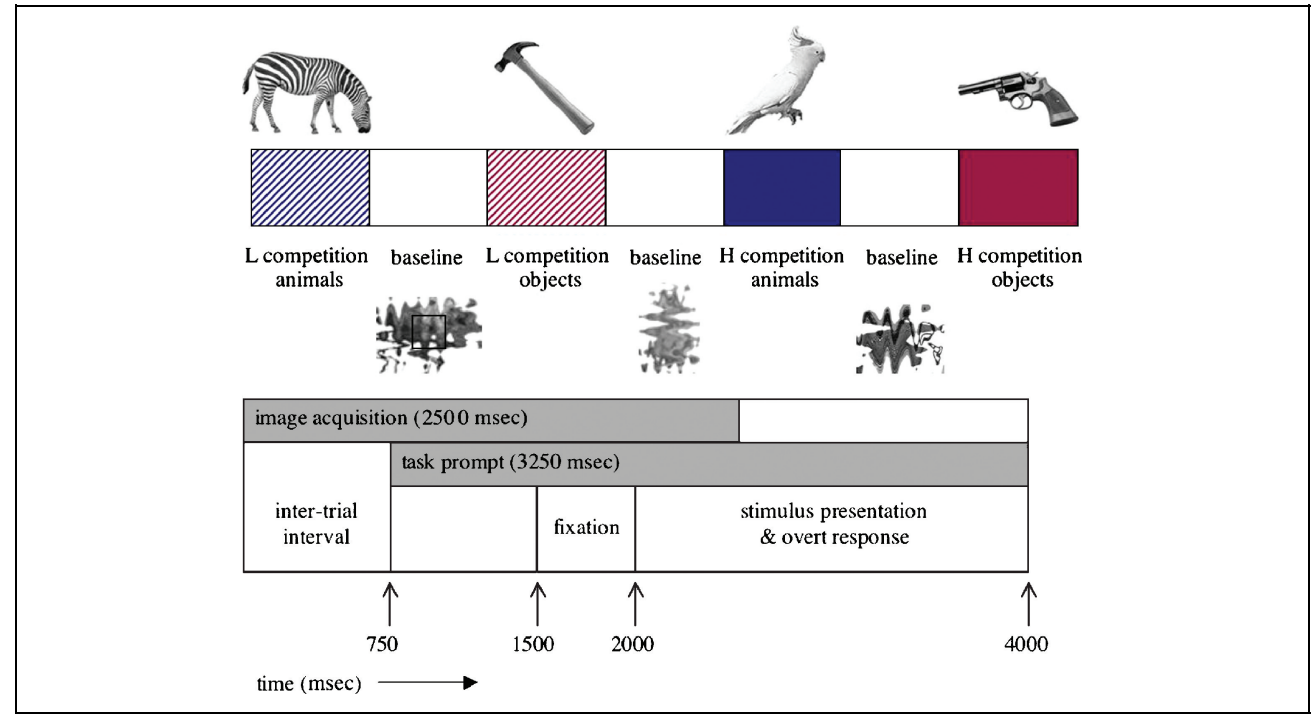

a significant difference between the low competition $(M=86.0 \%, S D=7.0 \%)$ and the high competition $(M=54.2 \%, S D=8.1 \%)$ conditions, $t(15)=19.36$, $p<.001$, confirming that the manipulation was effective.
Performance on baseline items derived from low competition photographs $(M=99.8 \%, S D=0.7 \%)$ was compared to performance on baseline items derived from high competition photographs $(M=99.8 \%, S D=$
Figure 2. Functionalanatomical ROI analysis. (A) Lateral projection of voxels demonstrating significant picture naming main effect (naming - baseline). Data were spatially smoothed for display purposes only. (B) Mixed ANOVA on mean variance normalized effect size in the left frontal operculum: significant competition main effect, $F(1,14)=10.34, p<.01$ and significant category main effect, $F(1,14)=4.71, p<.05$. Interaction was not significant. (C) Mixed ANOVA on mean variance normalized effect size in vLPM: significant category main effect $F(1,14)=13.03$, $p<.01$. Competition main effect and interaction were non-significant. Error bars indicate standard error of the mean effect size for each condition.

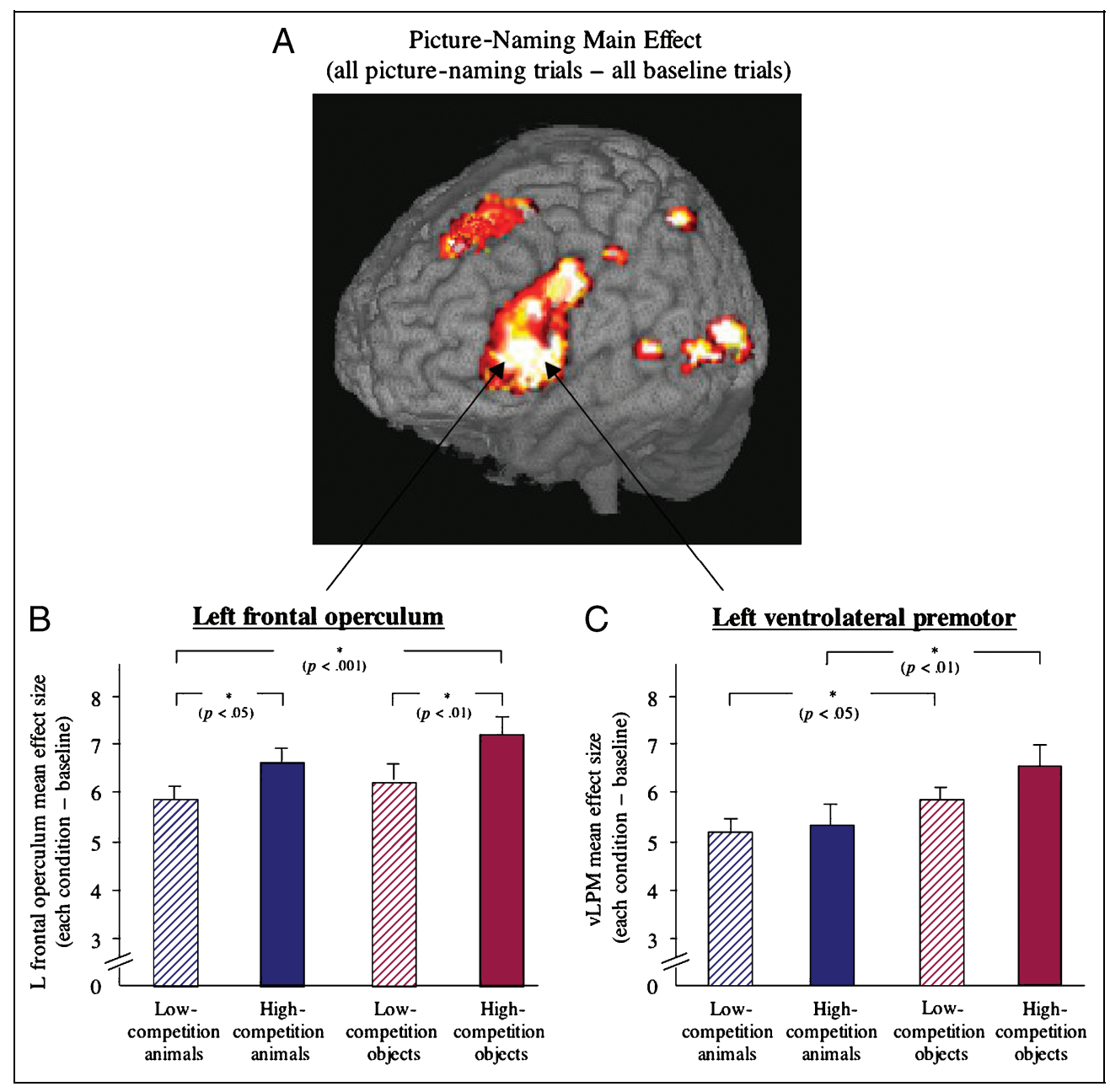


$0.5 \%)$, and no difference was found on response accuracy between the two baseline conditions, $t(15)=0.44$, $p>$.05. Due to technical constraints, however, we were unable to collect voice-activated response times from within the scanner.

\section{fMRI Results}

\section{Left Ventrolateral Premotor Region-of-Interest Analysis}

As expected, tool identification was associated with increased activity in the left frontal cortex; however, dissociable effects of motor knowledge retrieval and selection among competing alternatives were observed in distinct subregions of the inferior frontal gyrus. Within vLPM, we observed a significant object category main effect, with greater activity for manipulable objects than for animals, $F(1,14)=13.03, p<.01$ (see Figure 2C). Furthermore, the main effect of competition and higher order interactions were nonsignificant (all $p s>.10$ ). As discussed below, this pattern of data is in contrast to that found in the left frontal operculum.

\section{Left Frontal Operculum Region-of-Interest Analysis}

Within the left frontal operculum, we observed a significant main effect of competition, $F(1,14)=10.34, p<.01$. As predicted, we observed greater activity in the left frontal operculum for high competition items (i.e., low name agreement) than for low competition items (i.e., high name agreement), regardless of object category: interaction, $F(1,14)=0.50, p=.49$ (see Figure $2 \mathrm{~B}$ ). This finding is consistent with the idea that activity in the left frontal operculum is modulated by amount of competition when one must select an appropriate representation (e.g., Kan \& Thompson-Schill, 2004a; Nelson, ReuterLorenz, Sylvester, Jonides, \& Smith, 2003; ThompsonSchill, 2003; Thompson-Schill et al., 2002; Milham et al., 2001; Barch et al., 2000; Thompson-Schill et al., 1999; Thompson-Schill et al., 1998; Thompson-Schill et al., 1997) and that previously observed tool-specific response in this region may be partially driven by unintentional variation in picture name agreement between object categories. We also observed a significant main effect of category, $F(1,14)=4.71, p=$ .05 , in the left frontal operculum, with greater activity for manipulable objects than animals. To evaluate the relative magnitude of the category effect observed in the vLPM and the left frontal operculum, we conducted a 2 (category) $\times 2$ (region) ANOVA and found a significant ordinal interaction, suggesting that the magnitude of the category effect observed in vLPM is greater than that in the left frontal operculum, $F(1,15)=8.03, p=.01$ (see next paragraph for a related analysis).
Effect (Category vs. Competition) $\times$ Region (vLPM vs. Left Frontal Operculum) Analysis

As suggested by Sternberg (2001, 2004), to demonstrate separate modifiability of the two regions, we need to illustrate that each region is differentially sensitive to different experimental manipulations (i.e., category vs. competition). We examined the relative influence of the two processes (i.e., motor knowledge retrieval vs. selection among competing alternatives) by evaluating the relative magnitude of effects between the two experimental factors in the different brain regions. A significant Effect $\times$ Region interaction was found, $F(1,15)=27.18$, $p<.001$ (see Figure 3). Specifically, the competition effect (high competition - low competition) in the left frontal operculum was significantly larger than that in vLPM, $t(15)=3.14, p<.01$. In contrast, the category effect (objects - animals) was significantly larger in vLPM than in the left frontal operculum, $t(15)=2.99, p<.01$. This significant cross-over interaction indicates that the processes of motor knowledge retrieval and selection among competing alternatives have differential effects on vLPM and the left frontal operculum; following from Sternberg (2001, 2004), this pattern supports the idea that these two regions are functionally dissociable.

\section{Hand Preference in Object Manipulation and vLPM Activity}

To explore the relationship between individual motor experience and tool-specific activity in vLPM, we

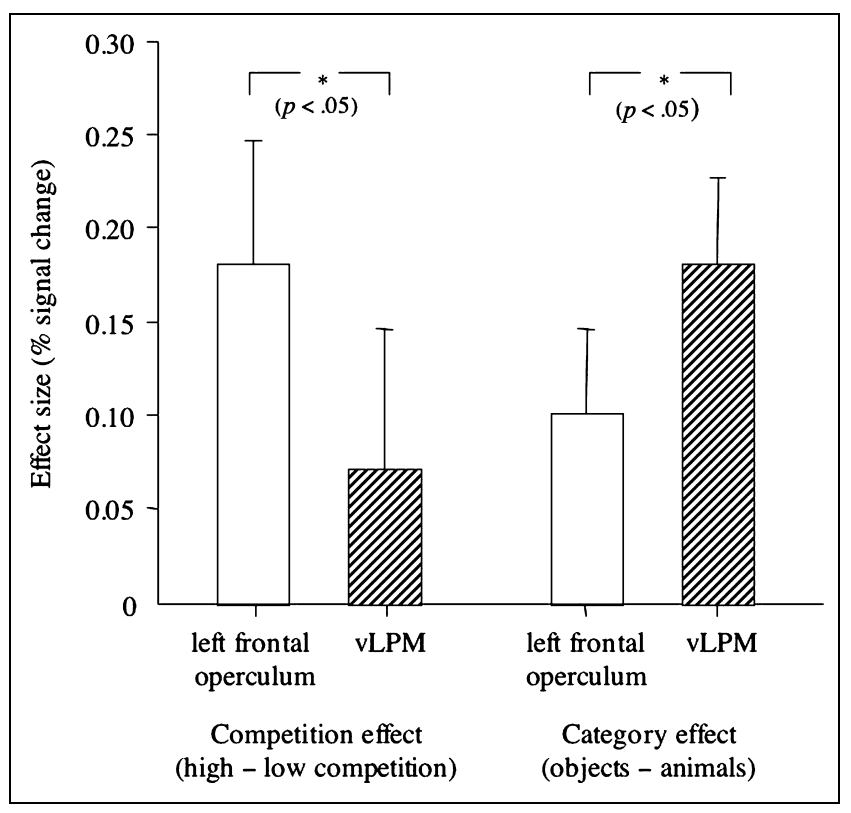

Figure 3. Effect $\times$ Region interaction bar plot. 2 (competition effect vs. category effect) $\times$ brain region (left frontal operculum vs. vLPM) ANOVA. Significant Effect $\times$ Region interaction was revealed using both percent signal change, $F(1,15)=13.37, p<.01$, and variance normalized effect size, $F(1,15)=27.18, p<.001$, as dependent measures. 
performed two analyses based on each subject's hand preference index and tool-specific activity in vLPM. As described in the Methods section, a "hand preference index" was derived for each subject, based on responses to items that were manipulated with only one hand. The score indicates the percentage of items each subject would prefer to manipulate with his right hand. Scores below 0.5 indicate a left-hand preference and scores above 0.5 indicate a right-hand preference when manipulating with objects named. Although right-handers showed a largely invariant right-hand preference (mean $=$ $.97, S D=.02$, range $=.95$ to 1.00$)$, left-handers' hand preference scores were much more variable (mean $=.23$, $S D=.27$, range $=.0$ to .72 ), and two left-handers showed a right-hand preference in object use.

A comparison of the magnitude of vLPM response between subjects with a left-hand preference to those with a right-hand preference (including the two "lefthanded" subjects who showed a right-hand preference) revealed greater vLPM activity in subjects with a righthand preference, $t(12)=2.19, p=.05$. Interestingly, no such difference in vLPM activity was found in a comparison of subjects based on handedness classification from the Edinburgh Handedness Inventory, $t(12)=-0.35$, $p=.73$, suggesting that our hand preference index may be a more sensitive measure of hand preference in object manipulation and that vLPM's response to these objects may be modulated by motor experience. The difference in sensitivity in these two measures is interesting, although not surprising. Whereas the Edinburgh Handedness Inventory serves as a good guideline for identifying hand preference, it is not a specific enough measure for evaluating an individual's hand preference in object manipulation.

Additional evidence for the claim that vLPM response may be modulated by motor experience comes from a correlational analysis of the tool-specific response in vLPM: In left-handers, the stronger the right-hand preference, the more robust the activity in $\operatorname{vLPM}(R=.88$, $p<.01$; see Figure 4). Given the small sample size, however, it is possible that outliers may exert undue influence over the observed effect. To address this concern, we also conducted the Spearman's rank order correlation test, a nonparametric correlation test that treats data points in terms of rank, thereby eliminating potential outlier effects. A significant positive correlation between vLPM activity and right-hand preference in object use was found $\left(R_{\mathrm{S}}=.81, p=.01\right)$. This pattern of data illustrates that vLPM activity is modulated by hand preference in object use and that this effect is specific to individuals' motor experience and not to a generalized handedness classification. In contrast to VLPM, the correlation between hand preference and activity in the left frontal operculum was not significant $\left(R=.56, p=.15 ; R_{\mathrm{S}}=.59, p=.13\right)$, thus providing additional support for the specific relationship between premotor cortex and motor experi-

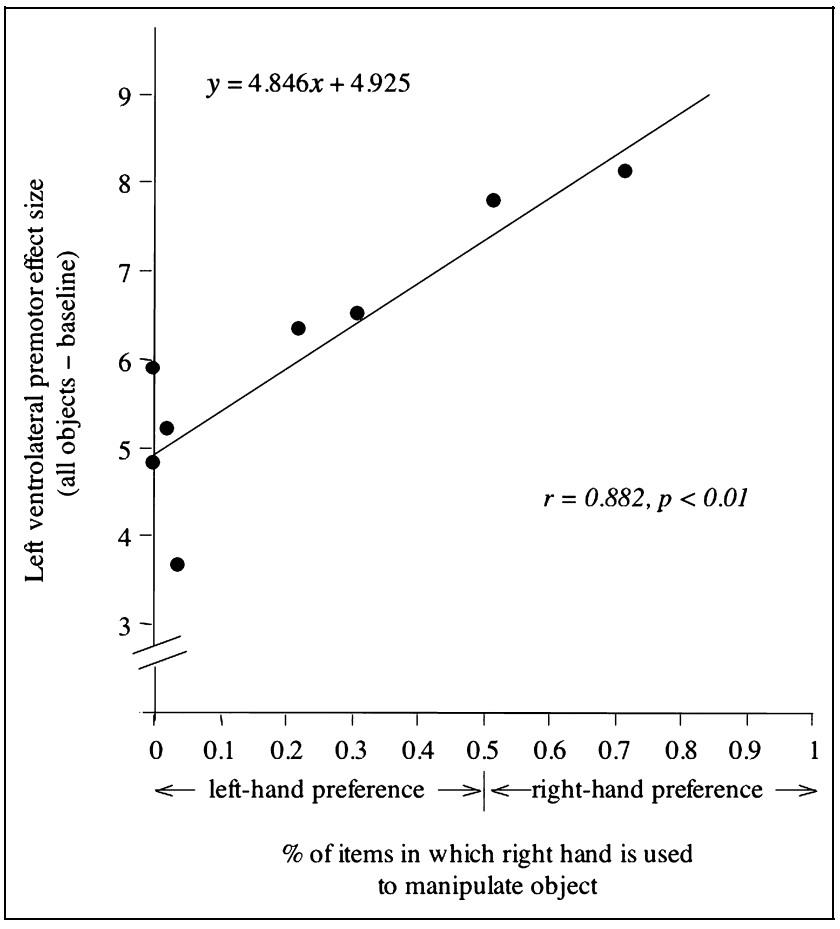

Figure 4. Scatterplot depicting the relationship between left-handers' hand preference in object use and object-related activity in vLPM.

ence. Furthermore, a direct comparison of the two correlations revealed that the magnitude of the correlation between hand preference in object use and vLPM activity is significantly larger than the magnitude of the correlation between hand preference in object use and activity in the left frontal operculum $\left(Z_{\mathrm{r}}=1.74\right.$, $p<.05)$. The largely invariant hand preference scores of right-handers precluded us from including their data in a correlational analysis (mean $=.97, S D=.02$ ).

In addition, there was no effect of hand preference on vLPM response to animals $(R=.32, p=.22)$. That is, hand preference did not result in a generalized pattern of lateralized or increased activity in VLPM, but only impacted vLPM's response to manipulable objects. Furthermore, activity in VLPM did not correlate with individuals' overall familiarity with the objects named $(R=.19, p=.32)$. Taken together, activity in the vLPM seems to be specific to manipulable objects (but not to animals) and is modulated by an individual's hand preference in object manipulation (but not by either general handedness classification or overall familiarity with objects).

Following from the idea that hand preference modulates object-related activity in the vLPM, one might also expect a positive correlation between left-hand preference and activity in the right ventrolateral premotor cortex (vRPM). However, we did not observe such a relationship ( $R=-0.23, p=.33$ ). Why might this asymmetry arise? One possible explanation is that perhaps "left-handers" have a more distributed motor 
representation than "right-handers," as suggested by previous studies on motor imagery (Singh et al., 1998). To explore this possibility, we derived a laterality index for each individual (vLPM activity - vRPM activity) and found a larger laterality effect for individuals with a right-hand preference than individuals with a lefthand preference, $t(10)=2.53, p=.03$. The same effect was found when a laterality ratio [i.e., (vLPM - vRPM)/ $(\mathrm{vLPM}+\mathrm{vRPM})]$ was used. (To derive a more precise characterization of the relationship between hand preference in object use and vLPM activity, it would be advantageous to evaluate these effects on an item-specific level. However, because a blocked design was employed, we are unable to examine item-specific effects in this experiment.)

\section{DISCUSSION}

In the present experiment, our aim was to (1) examine whether previously observed tool-specific activations in the left ventrolateral frontal cortex can be further dissociated and (2) explore the contribution of motor experiences to our neural and conceptual representations. Our data indicate that there are two distinct subpopulations of neurons within the left ventrolateral frontal cortex that have different response properties: an anterior region in the frontal operculum that responds to amount of competition present in object identification, and an adjacent premotor region that responds specifically to manipulable objects. Critically, we demonstrated functional dissociations in these regions by showing that these two regions are separately modifiable (Sternberg, 2001, 2004).

Our finding of selective influence of competition in the left frontal operculum activity provides converging evidence for the idea that the left frontal operculum mediates selection among competing alternatives. Specifically, in the context of object identification, we propose that activity in this region reflects the amount of lexical competition during picture naming (e.g., Kan \& Thompson-Schill, 2004a). This also lends support to the idea that discrepancy in previous experiments, in terms of the "tool-specific response" in the left frontal operculum, may be partially explained by unintentional variation in picture name agreement between manipulable objects and animals.

Consistent with previous studies (e.g., Tyler et al., 2003; Chao \& Martin, 2000; Perani et al., 1999; Grabowski et al., 1998; Grafton et al., 1997; Martin et al., 1996), we found that identification of manipulable objects engages vLPM, and recruitment of this region may reflect implicit retrieval of motor knowledge. One of the crucial assumptions of Allport's (1985) distributed model of conceptual knowledge is that conceptual representations are stored in or near brain regions that are used during initial encoding of that information. In other words, when action-oriented information is re- trieved, motor areas should be recruited. Based on this proposal, previous reports of left frontal activation during tool identification were taken as evidence for distributed models of concept representation in general and for a role of the premotor cortex in the representation of motor knowledge specifically (ThompsonSchill, 2003; Martin, 2001; Martin et al., 1996); however, those conclusions were based on the assumption that the frontal activation indicated motor knowledge retrieval. There was no independent source of evidence that the frontal activation was related to retrieval of motor knowledge per se. In the present study, the significant correlation between right-hand preference in object manipulation and left premotor activation during identification of manipulable objects may provide evidence for that missing link. In other words, our finding that an individual's hand preference in object use modulates object-related activity in vLPM provides additional support for domain-specific distributed models of conceptual knowledge (Thompson-Schill, 2003; Martin, 2001; Allport, 1985). Additionally, this domain-specific response was selective to vLPM and dissociable from a domain-general response in an adjacent ventrolateral prefrontal region.

Thus far, we have argued for and demonstrated a functional dissociation between vLPM and the left frontal operculum. However, within the left frontal operculum, we also observed a significant, albeit smaller, main effect of object category (i.e., objects $>$ animals) and a trend of a positive correlation between hand preference in object use and left frontal operculum activity. This pattern is consistent with previous findings that vLPM and the left frontal operculum often coactivate in tasks that require motor knowledge retrieval. Because the anatomical division between the two regions may not necessarily reflect a strict functional division of labor, it remains possible that the two regions represent a continuum. That is, although both regions may be involved in various processes of object identification, the posterior region of this continuum may be more sensitive to one process (i.e., motor knowledge retrieval) and the anterior region of this continuum may be more sensitive to another process (i.e., selection among competing alternatives). Of course, it is also possible that the seeming continuity of these effects reflects more about the properties of the fMRI signal (i.e., poor spatial resolution) than it does about the properties of the neurons within each region. In either case, the observation that the magnitude of the category effect and the correlation between hand preference and brain activity in the two regions are tightly coupled (i.e., larger category effect, higher correlation) suggests that the observed category effect is related to motor knowledge retrieval in object identification.

Why might these two adjacent regions subserve such seemingly different processes? One possibility is that perhaps the processes involved in selection and motor 
knowledge retrieval are both crucial to the development of human communication. In order to produce and comprehend language, one must select appropriate word meanings among competing alternatives. Moreover, a number of researchers (Rizzolatti \& Arbib, 1998; Bradshaw \& Rogers, 1993) have proposed that the ability to understand and recognize actions may be the foundation of the evolution of language (see Arbib, 2005, for a recent evolutionary account). For example, Rizzolatti and Arbib (1998) observed that neurons in monkey area F5, which corresponds to the inferior frontal cortex in the human brain, are involved in goal-related action execution (Rizzolatti et al., 1988) and action observation (Ferrari, Rozzi, \& Fogassi, 2005; Gallese, Fadiga, Fogassi, \& Rizzolatti, 1996); these F5 neurons within the action execution-recognition system were dubbed "mirror neurons." The authors argued that in order to react accordingly, it is essential for the observer to recognize the action itself and also the intention behind that action. From the agent's perspective, it is important to execute the action appropriately such that the agent's intention will not be misunderstood. Rizzolatti and Arbib (1998) proposed that this exchange of intentions forms the basis of communication. With the identification of a similar action recognition system in humans (Fadiga, Fogassi, Pavesi, \& Rizzolatti, 1995), the idea that both regions subserve the larger purpose of human communication seems plausible.

In summary, the proposal that sensorimotor experiences with manipulable objects influence neural representations is supported by the finding that hand preference in object manipulation is significantly correlated with activity in the vLPM. This finding is consistent with the proposal that organization of our conceptual knowledge is distributed across different domains as a function of our interaction with the physical world (Thompson-Schill, 2003; Martin, 2001; Allport, 1985). Furthermore, competition effects in the left frontal operculum support the idea of a domain-general selection mechanism (Thompson-Schill, 2003; ThompsonSchill et al., 2002). Taken together, this demonstration of the separate modifiability of activity in the vLPM and the left frontal operculum by different factors provides strong evidence that the two regions are associated with separable processes (Sternberg, 2001, 2004).

\section{METHODS}

\section{Behavioral Protocol}

Sixteen paid volunteers participated in this study: mean age $=21.0$ years $($ range $18-25$ years $) ; 8$ left-handers ( 4 men, 4 women, mean handedness score -12.40 ); 8 right-handers ( 2 men, 6 women, mean handedness score 18.6, range 15 to 22). The Edinburgh Handedness Inventory was used to assess handedness (Oldfield, 1971). In accordance with the procedures of the Insti- tutional Review Board of the University of Pennsylvania, all subjects provided informed consent, and each subject was paid $\$ 35$ for their participation.

\section{Stimulus Presentation}

Using PsyScope software (Cohen, MacWhinney, Flatt, \& Provost, 1993), stimuli were presented by a Macintosh G3 Powerbook (Apple Computer, Cupertino, CA) connected to an Epson 8100 3-LCD projector (Epson America, Long Beach, CA), which was housed inside a custom $\mathrm{RF}$ shield box. The image was projected onto a mylar rear projection screen at the back of the scanner bore, and subjects viewed the image through a mirror mounted on the head coil. Subjects' overt responses were transmitted via a microphone embedded in the Avotec Silent Scan Auditory Presentation system (Stuart, FL, www. avotec.org).

On each experimental trial, subjects were asked to say aloud the name of a black-and-white photograph of either an animal or a manipulable object as quickly as possible. Each trial lasted $4000 \mathrm{msec}$, and four components made up each trial (see Figure 1B for trial timing and composition). A total of 144 black-and-white photographs were used. Within each category (i.e., animals vs. objects), the photographs were further classified into conditions of high competition and low competition, which were determined by name agreement ratings derived from data collected from a group of independent subjects ( $n=32$, see Results section). Name agreement for each picture refers to the percentage of subjects who agree on a single name for each picture, and the data were used to facilitate stimulus selection. The variability in name agreement across subjects was used as a proxy measure of the degree of lexical competition within individuals.

On baseline trials, subjects were asked to perform a perceptual judgment task, which required a decision of whether a rectangular box was superimposed on top of a distorted image (see Figure 1A). Trial timing and composition were the same as the experimental trials. To create the baseline items, the set of 144 object and animal photographs were distorted by use of a distortion filter in Photoshop 7.0. Pilot testing indicated that the distorted photographs were no longer identifiable as real animals and objects.

A blocked design was implemented. Six trials composed each experimental block, and 16 blocks composed each experimental run. In each run, alternating blocks of picture-naming trials and baseline trials were presented, with 96 trials in each run: 12 highcompetition animals, 12 low-competition animals, 12 high-competition objects, 12 low-competition objects, and 48 baseline trials. Three experimental runs completed the experiment, for a total of 288 trials in the entire session. Presentation order of each experimental condition was counterbalanced across subjects. 
A postexperiment questionnaire was administered to examine the contribution of individual motor experience to neural representations of manipulable objects. For each object named in the experiment, subjects were asked to rate their hand preference when manipulating the object. Fourteen subjects completed the questionnaire ( 8 left-handers and 6 right-handers), and a hand preference index was derived for each subject, based on responses to items that were manipulated with only one hand. Two right-handers failed to complete the postexperiment questionnaire. Additionally, subjects were asked to report their overall familiarity with each item, which may include nonmotor experiences, such as seeing the item in books or on television. They were asked to rate their level of overall familiarity on a scale of 1 (not at all familiar) to 5 (extremely familiar).

\section{Functional MRI Protocol}

Following acquisition of sagittal and axial T1-weighted localizer images, echo-planar fMRI was performed in 42 contiguous 3-mm axial slices $(\mathrm{TR}=4000 \mathrm{msec}, \mathrm{TE}=$ $30 \mathrm{msec}, 64 \times 64$ pixels in a 24 -cm field of view, voxel size $=3 \mathrm{~mm} \times 3 \mathrm{~mm} \times 3 \mathrm{~mm})$, using a 3.0-T Siemens Trio system (Malvern, PA) and a USA Instruments (Aurora, $\mathrm{OH}$ ) four-channel head coil. Furthermore, 3-D prospective acquisition correction (PACE) was performed online during data acquisition, which allowed for realtime linear and rotational motion detection and correction (Siemens-Medical, 2004).

Offline data processing was performed using VoxBo software (www.voxbo.org). After image reconstruction, the data were sinc interpolated in time to correct for the staggered fMRI acquisition sequence. Motion detection and correction was undertaken using a six-parameter, rigid-body transformation. Consistent with previous findings (e.g., Kan \& Thompson-Schill, 2004a; Barch et al., 1999), overt responding did not lead to excessive motion artifacts, based on the amount of estimated movement occurred during each scan. None of the subjects had motion that resulted in more than a $2-\mathrm{mm}$ displacement. Raw data from each subject were transformed to standardized MNI space (Evans et al., 1993), and no spatial smoothing was performed. (Note: Spatial smoothing was performed for illustration purposes only, see Figure 2.)

Voxelwise analysis was performed on each subject's data by use of a general linear model for serially correlated error terms (Worsley \& Friston, 1995). The model included covariates that modeled the different task conditions, a subject-specific estimate of the intrinsic temporal autocorrelation, and sine and cosine regressors for frequencies below those of the task and those in the elevated range of the noise spectrum. Task covariates were boxcar waveforms convolved with an estimate of the bold oxygenation level dependent (BOLD) hemodynamic transfer function empirically derived from the motor cortex in a separate group of subjects (Aguirre, Zarahn, \& D'Esposito, 1998). Data were temporally smoothed with an empirically derived estimate of the hemodynamic response. These methods have been empirically demonstrated to hold the mapwise false-positive rate at or below tabular values (Aguirre, Zarahn, \& D'Esposito, 1997; Zarahn, Aguirre, \& D'Esposito, 1997).

We employed a functional-anatomical region-ofinterest (ROI) approach. Anatomical landmarks were used to define the two regions along the inferior frontal gyrus: The ventrolateral premotor cortex (inferior portion of BA 6) and the frontal operculum (BA 44/45) were defined for each individual subject. Each ROI consisted of voxels that demonstrated a significant picture-naming main effect (i.e., all picture-naming trials - baseline trials, $t>3.0)$. All voxels within each functionally-anatomically defined ROI were included in four contrast analyses between each experimental condition and baseline, and the magnitude of each contrast was estimated with a variance normalized measure of percent signal change (i.e., $t$ effect size). The magnitude of effect size for each contrast was entered into a 2 (competition) $\times 2$ (category) $\times 2$ (handedness) mixed analysis of variance.

\section{Acknowledgments}

We thank R. A. Epstein, S. Sternberg, and R. T. Oliver for helpful discussions and insightful comments on an earlier draft of the manuscript, and G. K. Aguirre, J. S. Higgins, and D. Y. Kimberg for assistance with data analysis. We also thank two anonymous reviewers for their helpful comments. This study was supported by grants from the National Institutes of Health (NIH R01 MH 60414 and NIH R01 MH 67008) and Searle Scholars Program (S. T. S), NIH Predoctoral Training Grant in Behavioral and Cognitive Neuroscience (I. P. K ), NSF graduate research fellowship (J. W. K), and NIH R01-DC04817 (A. C.).

Reprint requests should be sent to Irene P. Kan, Memory Disorders Research Center, Boston VA Healthcare System (151-A), 150 South Huntington Avenue, Jamaica Plain, MA 02130, or via e-mail: ikan@bu.edu.

The data reported in this experiment have been deposited in the fMRI Data Center (www.fmridc.org). The accession number is 2-2005-119FM.

\section{REFERENCES}

Aguirre, G. K., Zarahn, E., \& D’Esposito, M. (1997). Empirical analyses of BOLD fMRI statistics. II. Spatially smoothed data collected under null-hypothesis and experimental conditions. Neuroimage, 5, 199-212.

Aguirre, G. K., Zarahn, E., \& D'Esposito, M. (1998). The variability of human, BOLD hemodynamic responses. Neuroimage, 8, 360-369.

Allport, D. A. (1985). Distributed memory, modular subsystems and dysphasia. In S. K. Newman \& R. Epstein (Eds.), Current perspectives in dysphasia (pp. 207-244). Edinburgh: Churchill Livingstone.

Arbib, M. A. (2005). From monkey-like action recognition to human language: An evolutionary framework for neurolinguistics. Behavioral \& Brain Sciences, 28, 105-124. 
Barch, D. M., Braver, T. S., Sabb, F. W., \& Noll, D. C. (2000). Anterior cingulate and the monitoring of response conflict: Evidence from an fMRI study of overt verb generation. Journal of Cognitive Neuroscience, 12, 298-309.

Barch, D. M., Sabb, F. W., Carter, C. S., Braver, T. S., Noll, D. C., \& Cohen, J. D. (1999). Overt verbal responding during fMRI scanning: Empirical investigations of problems and potential solutions. Neuroimage, 10, 642-657.

Bradshaw, J. L., \& Rogers, L. (1993). The evolution of lateral asymmetries, language, tool use, and intellect. San Diego, CA: Academic Press.

Buckner, R. L., Koutstaal, W., Schacter, D. L., \& Rosen, B. R. (2000). Functional MRI evidence for a role of frontal and inferior temporal cortex in amodal components of priming. Brain, 123, Pt 3 620-640.

Bunge, S. A., Kahn, I., Wallis, J. D., Miller, E. K., \& Wagner, A. D. (2003). Neural circuits subserving the retrieval and maintenance of abstract rules. Journal of Neurophysiology, 90, 3419-3428.

Bunge, S. A., Ochsner, K. N., Desmond, J. E., Glover, G. H., \& Gabrieli, J. D. (2001). Prefrontal regions involved in keeping information in and out of mind. Brain, 124, 2074-2086.

Chao, L., \& Martin, A. (2000). Representation of manipulable man-made objects in the dorsal stream. Neuroimage, 12, 478-484.

Cohen, J. D., MacWhinney, B., Flatt, M., \& Provost, J. (1993). PsyScope: A new graphic interactive environment for designing psychology experiments. Behavioral Research Methods, Instruments, and Computers, 25, 257-271.

Decety, J. (1996). Do imagined and executed actions share the same neural substrate? Cognitive Brain Research, 3, 87-93.

Desmond, J. E., Gabrieli, J. D., \& Glover, G. H. (1998). Dissociation of frontal and cerebellar activity in a cognitive task: Evidence for a distinction between selection and search. Neuroimage, 7, 368-376.

Evans, A. C., Collins, D. L., Mills, S. R., Brown, E. D., Kelly, R. L., \& Peters, T. M. (1993). 3D statistical neuroanatomical models from 305 MRI volumes. Paper presented at the IEEE-Nuclear Science Symposium and Medical Imaging Conference, San Francisco, CA.

Fadiga, L., Fogassi, L., Pavesi, G., \& Rizzolatti, G. (1995). Motor facilitation during action observation: A magnetic stimulation study. Journal of Neurophysiology, 73, 2608-2611.

Ferrari, P. F., Rozzi, S., \& Fogassi, L. (2005). Mirror neurons responding to observation of actions made with tools in monkey ventral premotor cortex. Journal of Cognitive Neuroscience, 17, 212-226.

Gallese, V., Fadiga, L., Fogassi, L., \& Rizzolatti, G. (1996). Action recognition in the premotor cortex. Brain, 119, 593-609.

Grabowski, T. J., Damasio, H., \& Damasio, A. R. (1998). Premotor and prefrontal correlates of category-related lexical retrieval. Neuroimage, 7, 232-243.

Grafton, S. T., Fadiga, L., Arbib, M. A., \& Rizzolatti, G. (1997). Premotor cortex activation during observation and naming of familiar tools. Neuroimage, 6, 231-236.

Grezes, J., \& Decety, J. (2001). Functional anatomy of execution, mental simulation, observation, and verb generation of actions: A meta-analysis. Human Brain Mapping, 12, 1-19.

Grezes, J., Tucker, M., Armony, J., Ellis, R., \& Passingham, R. E. (2003). Objects automatically potentiate action: An fMRI study of implicit processing. European Journal of Neuroscience, 17, 2735-2740.

Hauk, O., Johnsrude, I., \& Pulvermuller, F. (2004). Somatotopic representation of action words in human motor and premotor cortex. Neuron, 41, 301-307.
Johnson-Frey, S. H. (2003). What's so special about human tool use? Neuron, 39, 201-204.

Kable, J. W., Lease-Spellmeyer, J., \& Chatterjee, A. (2002). Neural substrates of action event knowledge. Journal of Cognitive Neuroscience, 14, 795-805.

Kan, I. P., \& Thompson-Schill, S. L. (2004a). Effect of name agreement on prefrontal activity during overt and covert picture naming. Cognitive, Affective and Behavioral Neuroscience, 4, 43-57.

Kan, I. P., \& Thompson-Schill, S. L. (2004b). Selection from perceptual and conceptual representations. Cognitive, Affective \& Behavioral Neuroscience, 4, 466-482.

Kellenbach, M. L., Brett, M., \& Patterson, K. (2003). Actions speak louder than functions: The importance of manipulability and action in tool representation. Journal of Cognitive Neuroscience, 15, 30-46.

Martin, A. (2001). Functional neuroimaging of semantic memory. In R. Cabeza \& A. Kingstone (Eds.), The bandbook of functional neuroimaging (pp. 153-186). Cambridge: MIT Press.

Martin, A., Haxby, J. V., Lalonde, F. M., Wiggs, C. L., \& Ungerleider, L. G. (1995). Discrete cortical regions associated with knowledge of color and knowledge of action. Science, 270, 102-105.

Martin, A., Wiggs, C. L., Ungerleider, L. G., \& Haxby, J. V. (1996). Neural correlates of category-specific knowledge. Nature, 379, 649-651.

Milham, M. P., Banich, M. T., Webb, A., Barad, V., Cohen, N. J., Wszalek, T., \& Kramer, A. F. (2001). The relative involvement of anterior cingulate and prefrontal cortex in attentional control depends on nature of conflict. Cognitive Brain Research, 12, 467-473.

Nelson, J. K., Reuter-Lorenz, P. A., Sylvester, C.-Y. C., Jonides, J., \& Smith, E. E. (2003). Dissociable neural mechanisms underlying response-based and familiarity-based conflict in working memory. Proceedings of the National Academy of Sciences, U.S.A., 100, 11171-11175.

Oldfield, R. (1971). The assessment and analysis of handedness: The Edinburgh Inventory. Neuropsychologia, 9, 97-113.

Perani, D., Schnur, T., Tettamanti, M., 'Gorno-Tempini, M., Cappa, S. F., \& Fazio, F. (1999). Word and picture matching: A PET study of semantic category effects. Neuropsychologia, 37, 293-306.

Phillips, J. A., Noppeney, U., Humphreys, G. W., \& Price, C. J. (2002). Can segregation within the semantic system account for category-specific deficits? Brain, 125, 2067-2080.

Pulvermuller, F. (1999). Words in the brain's language. Behavioral \& Brain Sciences, 22, 253-279; discussion 280-336.

Rizzolatti, G., \& Arbib, M. A. (1998). Language within our grasp. Trends in Neurosciences, 21, 188-194.

Rizzolatti, G., Camarda, R., Fogassi, L., Gentilucci, M., Luppino, G., \& Matelli, M. (1988). Functional organization of inferior area 6 in the macaque monkey. II. Area F5 and the control of distal movements. Experimental Brain Research, 71, 491-507.

Rizzolatti, G., Fogassi, L., \& Gallese, V. (2002). Motor and cognitive functions of the ventral premotor cortex. Current Opinions in Neurobiology, 12, 149-154.

Siemens-Medical. (2004). Motion under control with prospective acquisition correction (PACE). Malvern, PA: Siemens Medical.

Singh, L. N., Higano, S., Takahashi, S., Kurihara, N., Furuta, S., Tamura, H., Shimanuki, Y., Mugikura, S., Fujii, T., Yamadori, A., Sakamoto, M., \& Yamada, S. (1998). Comparison of ipsilateral activation between right and 
left handers: A functional MR imaging study. NeuroReport, 9, 1861-1866.

Sternberg, S. (2001). Separate modifiability, mental modules, and the use of pure and composite measures to reveal them. Acta Psychologica, 106, 147-246.

Sternberg, S. (2004). Separate modifiability and the search for processing modules. In N. G. Kanwisher \& J. Duncan (Eds.), Attention and performance XX: Functional brain imaging of visual cognition. New York: Oxford University Press.

Tettamanti, M., Buccino, G., Saccuman, M. C., Gallese, V., Danna, M., Scifo, P., Fazio, F., Rizzolatti, G., Cappa, S. F., \& Perani, D. (2005). Listening to action-related sentences activates fronto-parietal motor circuits. Journal of Cognitive Neuroscience, 17, 273-281.

Thompson-Schill, S. L. (2003). Neuroimaging studies of semantic memory: Inferring "how" from "where." Neuropsychologia, 41, 280-292.

Thompson-Schill, S. L., D'Esposito, M., Aguirre, G. K., \& Farah, M. J. (1997). Role of left inferior prefrontal cortex in retrieval of semantic knowledge: A reevaluation. Proceedings of the National Academy of Sciences, U.S.A., 94, 14792-14797.

Thompson-Schill, S. L., D'Esposito, M., \& Kan, I. P. (1999). Effects of repetition and competition on prefrontal activity during word generation. Neuron, 23, 513-522.

Thompson-Schill, S. L., Jonides, J., Marshuetz, C., Smith, E. E.,
D'Esposito, M., Kan, I. P., Knight, R. T., \& Swick, D. (2002). Effects of frontal lobe damage on interference effects in working memory. Cognitive, Affective and Behavioral Neuroscience, 2, 109-120.

Thompson-Schill, S. L., Swick, D., Farah, M. J., D'Esposito, M., Kan, I. P., \& Knight, R. T. (1998). Verb generation in patients with focal frontal lesions: A neuropsychological test of neuroimaging findings. Proceedings of the National Academy of Sciences, U.S.A., 95, 15855-15860.

Tyler, L. K., Stamatakis, E. A., Dick, E., Bright, P., Fletcher, P., \& Moss, H. (2003). Objects and their actions: Evidence for a neurally distributed semantic system. Neuroimage, 18 , 542-557.

Vitali, P., Abutalebi, J., Tettamanti, M., Rowe, J., Scifo, P., Fazio, F., Cappa, S. F., \& Perani, D. (2005). Generating animal and tool names: An fMRI study of effective connectivity. Brain and Language, 93, 32-45.

Wagner, A. D., Pare-Blagoev, E. J., Clark, J., \& Poldrack, R. A. (2001). Recovering meaning: Left prefrontal cortex guides controlled semantic retrieval. Neuron, 31, 329-338.

Worsley, K. J., \& Friston, K. (1995). Analysis of fMRI time-series revisited-Again. Neuroimage, 2, 173-182.

Zarahn, E., Aguirre, G. K., \& D'Esposito, M. (1997). Empirical analyses of BOLD fMRI statistics. I. Spatially unsmoothed data collected under null-hypothesis conditions. Neuroimage, 5, 179-197. 